\title{
Abordaje psicoeducativo para estimular la atención y las habilidades interpersonales en escolares argentinos*
}

\author{
Mirta Susana Ison** \\ Universidad del Aconcagua, Mendoza (Argentina)
}

Recibido: 19 de abril del 2009 / Aprobado: 20 de julio del 2009

Los objetivos del presente trabajo son: a) identificar a escolares entre los 7 y 12 años de edad, de ambos géneros, que presenten disfunción en atención sostenida $(D A S), b)$ explorar la memoria de trabajo y las habilidades cognitivas para la solución de problemas interpersonales en los escolares con DAS, y c) demostrar la efectividad de programas de intervención en los niños participantes.

disfunción atencional en niños / habilidades sociocognitivas /

programas de intervención

\section{Psychoeducational approach to stimulate attentional functioning and} interpersonal abilities in Argentinean school students

In this article we focused: a) to identify students, within both gender ranges and between 7 and 12 years old, with sustained attention dysfunction (SAD), b) to explore the working memory and the cognitive skills necessary for interpersonal problem solving in students identifies as $S A D$, and c) to demonstrate the effectiveness of the intervention programs in these children.

attentional dysfunction in children / socio-cognitive skills in children / intervention programs

Correo electrónico: mison@mendoza-conicet.gov.ar

* Este artículo forma parte del proyecto de la Carrera de Investigador Científico y Tecnológico dependiente del Conicet, PIP 5207. Subsidiado parcialmente por la Universidad del Aconcagua a través del Proyecto PS 06 Psicología. Mendoza- Argentina.

** Agradezco a mi equipo de trabajo la colaboración incondicional en los proyectos de investigación realizados. Dicho equipo está conformado por profesionales, becarias de Conicet y profesoras de la Universidad del Aconcagua: Gabriela Morelato, Mariana Carrada, Silvina Maddio, Carolina Greco y María Elsa Porta, y por docentes investigadoras de la Universidad del Aconcagua: Adriana Espósito y Celina Korzeniowski. 


\section{INTRODUCCIÓN}

El presente trabajo es fruto de la experiencia lograda luego de la ejecución de diferentes proyectos de investigación realizados desde el año 2002 hasta la fecha, en escuelas estatales de la provincia de Mendoza, Argentina.

Estas investigaciones persiguieron dos grandes objetivos: el primero fue implementar estrategias de intervención tendientes a fortalecer los recursos atencionales y las habilidades sociales y cognitivas para solucionar problemas interpersonales. Dichas estrategias fueron especialmente diseñadas sobre la base de una evaluación diagnóstica cuyo objetivo fue detectar disfunciones atencionales y en las habilidades cognitivas de solución de problemas interpersonales. El segundo objetivo fue conocer cuán eficaz resultaba el programa de intervención aplicado para el fortalecimiento y la recuperación de las funciones previamente evaluadas. Vale decir, como equipo de investigación no solo nos interesa diseñar y aplicar programas de intervención sino también probar su eficacia.

En las escuelas de la provincia de Mendoza los comportamientos agresivos, los impulsos, el retraimiento social, la hiperactividad y las disfunciones atencionales (Ison, 2001a; 2001b) son factores de riesgo significativos para el retraso escolar. Mientras la hiperactividad tiende a disminuir con la edad, algunos comportamientos agresivos-impulsivos y, sobre todo, la dificultad para sostener la atención se mantienen como dificultades sobresalientes en muchos casos (Bauermeister, 2002).

\section{LA ATENCIÓN Y SU RELACIÓN CON EL DESEMPEÑO ESCOLAR}

La atención es definida como un mecanismo "vertical de control", cuya función no es tanto elaborar y procesar información como regular y dirigir los mecanismos responsables del procesamiento, activando o inhibiendo los procesos encargados de elaborar y organizar la información (Tudela, 1992; Roselló i Mir, 1998).

Para responder con cierto grado de eficacia a las demandas cambiantes del ambiente, el sujeto debe disponer de recursos atencionales que le permitan dirigir la acción hacia las diferentes metas propuestas. Dependiendo de las características de la tarea y del contexto, el sujeto tendrá que ejercer un mayor o menor control atencional. Actualmente, el control atencional es considerado como un requisito indispensable para el funcionamiento del sistema ejecutivo (SE). Este se pone en marcha frente a situaciones en las que el sujeto debe realizar una acción no rutinaria, encaminada a la consecución de un objetivo que exige inhibir las respuestas habituales, requiere planificación de las conductas y toma de decisiones y, para ello, precisa de la atención consciente (Anderson, 2002; Sánchez Carpintero \& Narbona, 2004). 
El escolar, para conseguir los objetivos curriculares mínimos, debe estar lo suficientemente motivado para el aprendizaje de nuevos contenidos. Para ello es necesario contar con recursos atencionales que le permitan poner en marcha las diferentes funciones ejecutivas. Ellas son: a) planificación de la conducta dirigida a una meta, b) programación de acciones necesarias para lograr dicha meta, c) monitorización de la puesta en marcha del plan para comprobar su ajuste al objetivo y estrategias iniciales, d) control de la interferencia de estímulos externos irrelevantes al plan de acción, e) flexibilidad cognitiva para corregir errores o generar nuevas conductas en función de las demandas del ambiente, y f) finalización de la acción cuando los objetivos se hayan cumplido (SánchezCarpintero \& Narbona, 2004). Es decir, el escolar debe disponer eficazmente de las habilidades para focalizar, distribuir y mantener su atención durante el tiempo que le requiera la tarea. Estas habilidades atencionales permiten a la memoria de trabajo almacenar y disponer temporalmente de la información con el fin de planificar y organizar estratégicamente esos contenidos.

La memoria de trabajo se define como un mecanismo de almacenamiento temporal que permite retener algunos datos de información, compararlos, contrastarlos o, en su lugar, relacionarlos entre sí. Se ocupa del almacenamiento de información a corto plazo, a la vez que manipula información necesaria para las habilidades cognitivas más complejas (Baddeley, 1999). Una clase especial de estas habilidades son aquellas implicadas en la solución cognitiva de problemas interpersonales.

Otro aspecto para tener en cuenta es el desarrollo evolutivo de la atención y las funciones ejecutivas. El mecanismo atencional se desarrolla progresivamente conforme con las distintas etapas evolutivas del individuo. A los 7 años el rendimiento en tareas de sostenimiento atencional mejora, en relación con edades anteriores, y a los 8-9 años se produce una mejora crítica, para estabilizarse alrededor de los 17 años (García Sevilla, 1997). Por su parte, las investigaciones realizadas sobre el desarrollo evolutivo de las funciones ejecutivas sostienen que entre los 7 y los 10 años de edad es el período donde se produce el mayor avance (Romero-Ayuso, Maestú, González-Marqués, RomoBarrientos, Andrade, 2006).

Por consiguiente, el mecanismo atencional estaría encaminado a conseguir un control de la cognición y la acción del organismo, sobre todo ante situaciones novedosas y ante situaciones que requieren actividades cuidadosamente planificadas y organizadas (Roselló i Mir, 1998). Dicho de otro modo, los niveles satisfactorios de atención sostenida son imprescindibles para la puesta en marcha de las funciones ejecutivas. 
Sobre la base de lo anterior, para que un escolar pueda tener un desempeño académico satisfactorio debe desarrollar una serie de habilidades sociales, afectivas y cognitivas. Estas capacidades se van desarrollando en una estrecha interrelación entre los procesos madurativos $\mathrm{y}$ las posibilidades de aprendizaje que el contexto social le ofrece.

Familia, escuela y grupo de pares conforman los principales contextos del desarrollo infantil y proporcionan al niño/a un marco de referencia para su actuación social al validar, rectificar o desaprobar las conductas realizadas por este en situaciones de interacción social. Por consiguiente, el niño internaliza, interpreta y responde a las demandas y restricciones provenientes de los diferentes agentes socializantes. De este modo, comienza a adquirir y a consolidar determinados esquemas cognitivos-afectivos que sustentan las habilidades interpersonales (Ison \& Morelato, 2008).

Frente a contextos con elevadas condiciones de vulnerabilidad social existe mayor probabilidad de que los niños presenten dificultades en su desarrollo cognitivo, afectivo y social (Ison, 2004; Lipina, Martelli, Vuelta, Injoque-Ricle \& Colombo, 2004; Musso, 2005; Richaud de Minzi, 2007) y esto se vea reflejado en el desempeño académico y social del escolar. Por consiguiente, las habilidades cognitivas para afrontar y resolver problemas interpersonales es otro de los compo- nentes que se incluyen en los programas de intervención.

\section{Habilidades cognitivas para la SOLUCIÓN DE PROBLEMAS INTERPERSONALES EN ESCOLARES}

En el dinámico proceso de enseñanzaaprendizaje, el escolar debe afrontar un sinnúmero de situaciones, muchas de las cuales pueden representar un problema. Un problema se presenta cuando la persona no sabe qué serie de acciones llevar a cabo para arribar a la solución (Newell \& Simon, 1972, citado por Zelazo et al., 1997). Frente a un conflicto interpersonal pueden utilizarse estrategias impulsivas y/o agresivas o bien apelar al afrontamiento reflexivo de la situación. Para este último caso, el escolar deberá desarrollar paulatinamente la flexibilidad cognitiva, la cual permitirá: a) reconocer que el problema puede ser resuelto, b) identificar la situación problema y definirla, es decir elaborar un mapa mental o representación interna del problema, c) planificar y organizar su conducta en función del objetivo propuesto, es decir pensar diversas posibilidades y estrategias para su solución previendo las consecuencias, d) monitorizar la puesta en marcha del plan de acción para evaluar las estrategias utilizadas en relación con el objetivo, e) elegir la solución más apropiada mediante un proceso de toma de decisiones, y f) evaluar la ejecución del plan para determinar si 
el problema ha sido resuelto de manera efectiva o se deben generar nuevas conductas en función de los resultados obtenidos (Ison, 2006; Ison, Espósito, Carrada, Morelato, Maddio, Greco \& Korzeniowski, 2007; Ochoa Angrino, Cruz Panesso \& Valencia, 2006; Sánchez-Carpintero \& Narbona, 2004).

Para que los niños comprendan estos pasos y puedan poner en práctica las habilidades cognitivas de solución de problemas de modo efectivo se requiere ciertas condiciones previas: motivación para que la memoria pueda retener lo aprendido, toma de perspectiva social, autorregulación emocional y esfuerzo para mantener la atención. Investigaciones realizadas por nuestro equipo muestran que los niños con disfunción atencional utilizan estrategias de resolución de problemas poco eficaces a la hora de solucionar problemas interpersonales (Ison, Morelato, Casals, Maddio, Carrada, Espósito, Greco \& Arrigoni, 2005).

Diversas investigaciones muestran la importancia de la estimulación socioambiental sobre el desarrollo cerebral infantil. Se sabe que la estimulación sensorial temprana estabiliza los circuitos cerebrales encargados del aprendizaje y del almacenamiento de la información. De este modo, padecer de emprobrecimiento sensorial puede dejar una impronta en el cerebro (Chugani, 1998). En los últimos años, el avance de las neurociencias cognitivas y de los estudios por imagen permiten identifi- car con mayor precisión diferentes redes neuronales implicadas en diversos procesos cognitivos y afectivos complejos (Pistoia, Abad-Mas \& Etchepareborda, 2004). Desde un punto de vista funcional puede afirmarse que la corteza prefrontal es el asiento de las funciones cognitivas más complejas y evolucionadas del ser humano, como lo son la resolución de problemas, la conducta social, el juicio ético-moral, la expresión de emociones positivas y negativas o la conducta creativa, entre otras (Muñoz-Céspedes \& TirapuUstárroz, 2004).

En la actualidad, la literatura científica habla de los beneficios asociados a los abordajes multimodales en el tratamiento de las diversas problemáticas infantiles (Gauvain \& Huard, 1999; Ison, 2006; NICHD, 2005; RecartHerrera, Mathiesen-De Gregori \& Herrera-Garbarini, 2005). Así las funciones sociocognitivas disminuidas pueden ser recuperadas por medio de la aplicación de programas específicos de intervención, a través de los cuales se desarrollan habilidades cognitivas, afectivas y sociales que contribuyen a mejorar el desempeño de un individuo en diversas actividades (Pistoia et al., 2004; Muñoz-Céspedes \& TirapuUstárroz, 2004; Ison et al., 2005; Ison et al., 2007). A su vez, las intervenciones que tengan como fin estimular las funciones cognitivas con el propósito de mejorar el desempeño académico y socioemocional del niño deberán in- 
cluir a los padres y docentes como agentes centrales de intervención en el proceso de aprendizaje escolar.

Desde el año 2000, el equipo que dirijo ha realizado diversas investigaciones tendientes a evaluar la atención y las habilidades cognitivas para la solución de problemas interpersonales en escolares mendocinos y, sobre estos resultados, diseñar e implementar estrategias de intervención tendientes a potenciar y favorecer el desarrollo de las funciones mediante el enriquecimiento de los recursos cognitivo-afectivos de los niños que crecen en situación de vulnerabilidad social. Estas habilidades cognitivas sustentan el aprendizaje escolar en el niño (Ison, 2004, 2006, 2007; Ison et al., 2005; Ison et al., 2007). Este abordaje tiene en cuenta las características del contexto psicosocial del niño y de su familia, integrando a los programas de intervención a cuidadores, padres y docentes como agentes centrales en el proceso de aprendizaje escolar.

Programa de INTERVENCIÓN PARA ESTIMULAR UNA ATENCIÓN SOSTENIDA Y HABILIDADES SOCIOCOGNITIVAS EN ESCOLARES ARGENTINOS: ABORDAJE Psicoeducativo

La estimulación neurocognitiva a través de programas por ordenador en combinación con otras estrategias de intervención demuestran ser herramientas indispensables para la rehabili- tación y entrenamiento de las funciones neuropsicológicas específicas. Este es el fundamento de los programas de intervención para el desarrollo de la capacidad atencional y las funciones ejecutivas en niños con déficits en los mecanismos reguladores de la atención (Ison, 2004; Ison et al., 2005; Pistoia, Abad-Mas \& Etchepareborda, 2004).

Desde el año 2002 estamos aplicando un programa de intervención a escolares con disfunción atencional en escuelas estatales de la provincia de Mendoza, con el objetivo de potenciar los recursos atencionales y fortalecer las habilidades cognitivas para resolver problemas interpersonales. El fundamento de este programa se basa en comprender que la disfunción atencional, presentada por el escolar, debe ser abordada teniendo en cuenta el interjuego que se produce entre el niño, el contexto del aula y su familia.

Se trabaja con niños en edad escolar del primer y segundo ciclos de la educación general básica. El establecimiento educativo debe prestar su consentimiento para el desarrollo del programa de intervención y se informa a los docentes y padres acerca de los objetivos de este con el fin de obtener su colaboración y autorización para que los escolares participen en el programa de intervención.

Las estrategias de intervención son dirigidas hacia el escolar y hacia el contexto de interacción social próximo. 
- Intervención centrada en el escolar

- Test de atención infantil 1.0. Programa computarizado para el entrenamiento de la atención (Ison, Soria \& Ana, 2003). Este programa fue diseñado para mejorar la capacidad de atención sostenida y la discriminación perceptiva. Fue elaborado en el Instituto de Ciencias Humanas Sociales y Ambientales (Incihusa-Conicet-CCT) y utilizado en las investigaciones realizadas desde el 2002 hasta la fecha.

El Test de Atención Infantil 2.0 consta de distintos subtest dirigidos a estimular la focalización, el sostenimiento atencional y discriminación perceptual mediante tareas de búsqueda visual. A su vez, cada subtest consta de sesiones de entrenamiento con el fin de cerciorarse de que la consigna haya sido adecuadamente comprendida por el escolar y para familiarizarlo con el programa. En esta instancia el investigador puede ayudar, orientar y explicar al escolar lo que sea necesario para que pueda realizar la segunda fase, que es la del test propiamente dicho. Para cada uno de los subtest existen distintos parámetros que aumentan o disminuyen el nivel de complejidad de estos y pueden ser modificados por el investigador. En las sesiones de entrenamiento el investigador explicaba al niño la consigna de la tarea, luego el niño practica unos minutos hasta que el investigador corrobora que la consigna ha sido comprendida por el niño. Posteriormente, el niño realiza el ejercicio en forma individual (fase de test). En la fase de test $\mathrm{el}$ programa registra el número de aciertos, errores y omisiones, como también el tiempo total de realización de la prueba.

- Programa para el desarrollo de habilidades cognitivas para la solución de problemas interpersonales (Ison, Morelato, Greco \& Maddio, 2006)

El programa para estimular las habilidades cognitivas para la solución de problemas interpersonales fue elaborado por nuestro equipo de investigación (Ison, Morelato, Greco, Maddio \& Korzeniowski, 2006) para promover la identificación y descripción de situaciones cotidianas que pudieran representar un problema en un contexto de interacción social, para estimular el reconocimiento de las emociones asociadas al problema, la generación de alternativas de solución a los problemas identificados y la anticipación de consecuencias y, finalmente, para discriminar aquellas alternativas conducentes a la elección de soluciones asertivas que impli- 
quen mayores beneficios y menores costos. Esto involucra el proceso de toma de decisiones acertadas el cual depende, en muchos casos, del análisis de las distintas posibilidades para su solución y del análisis de las consecuencias de cada una de esas alternativas. Tal como sostiene Bornas (1998), tomar decisiones adecuadas permite emprender la tarea con mayores posibilidades de éxito.

Las temáticas trabajadas fueron: a) descripción de situaciones cotidianas que representan un problema; b) reconocimiento de emociones asociadas a ese problema; c) búsqueda de soluciones, y d) toma de decisión evaluando cuál es la mejor alternativa y por qué.

Se trabajó con pequeños grupos en los cuales el experto explicaba a los escolares la actividad por realizar en cada encuentro, brindaba material de trabajo, feedback, y al final de cada encuentro, se realizaba un resumen y revisión del módulo.

- Centrado en el contexto de interacción social

Las estrategias de intervención dirigidas hacia el contexto de interacción social próximo se realizaron a través de talleres de capacitación a docentes y padres. Estos tienen como propósito central brindar información integral sobre la problemática para que padres y docentes puedan asumir un rol activo en el acompañamiento y estimulación de los niños. Se trata de promover un cambio de actitud frente a la problemática del niño $\mathrm{y}$, al mismo tiempo, entrenar a estos adultos en uso de habilidades y estrategias efectivas para abordar las dificultades de los niños.

Un niño con disfunción ejecutiva presenta un patrón de conductas, habilidades y cogniciones diferente al de los niños sin esta problemática y por eso requiere estrategias y pautas educativas particulares. Generalmente, los adultos responsables de su educación no disponen de dichos conocimientos y estrategias, resultándoles difícil comprender y manejar la particularidad del pequeño. En la mayoría de los casos, esta situación ocasiona dificultades familiares y escolares significativas, las cuales repercuten negativamente en el desarrollo integral del niño. Por este motivo, capacitar a padres y docentes es una tarea indispensable si se desea realizar un abordaje integral de la problemática.

- Taller para padres.- Su finalidad fue brindar información científica actualizada sobre la disfunción atencional y su repercusión en el aprendizaje escolar, con el objetivo de desarrollar en los 
padres habilidades cognitivas necesarias para la resolución de conflictos interpersonales y estrategias de intervención específicas para disminuir la conducta inatenta en sus hijos. Así, se trabajaron estos temas para mejorar la calidad de las interacciones entre padres e hijos, promoviendo el respeto mutuo, la cooperación y la confianza básica.

El taller fue estructurado en seis encuentros grupales, que se realizaron una vez por mes. Cada encuentro incluyó objetivos, actividades y tareas específicas.

En el primer encuentro se brindó información científica y actualizada sobre la disfunción atencional para facilitar a los padres la comprensión de la problemática y la corrección de mitos e ideas erróneas preexistentes. En el segundo encuentro se identificaron los factores que influyen en la aparición y mantenimiento de las dificultades atencionales y se trabajaron estrategias específicas para incrementar la capacidad atencional en el escolar. En el tercer encuentro se indicó a los padres los ejercicios específicos que debían implementar para el desarrollo de la organización y planificación de las tareas escolares en sus hijos. En el cuarto y quinto encuentros se trabajaron las habilidades cognitivas para la resolu- ción de conflictos interpersonales, identificando las situaciones problema, las emociones asociadas a ellas, la generación de alternativas, la anticipación de consecuencias y la toma de decisiones. Finalmente, en el último encuentro se abordó cómo afrontar futuras dificultades en los escolares y después los padres realizaron la evaluación final del taller.

- Talleres para docentes.- Fueron organizados en cuatro encuentros grupales bimestrales; además, se trabajó individualmente con el docente en el aula. $\mathrm{Su}$ finalidad fue la de brindar información sobre la disfunción atencional, facilitar ejercitación específica para el docente y diseñar estrategias para el manejo efectivo de la conducta inatenta y sus dificultades asociadas con el fin de que el docente las implemente en el aula. Por su parte, el trabajo individual con el docente consistió en brindarle asesoramiento sobre el modo de implementar las estrategias con el grupo de alumnos.

En el primer encuentro se abordaron los factores que influyen negativamente en el rendimiento académico y en la adaptación escolar del niño con disfunción atencional. Además, se reflexionó sobre las dificultades y fortalezas de los niños con esta 
problemática. En el segundo encuentro se trató acerca de cómo obtener y mantener la atención y motivación de un niño con disfunción atencional en el aula. En el tercer encuentro se trabajó con los docentes las habilidades cognitivas para la resolución de conflictos interpersonales, y en el último encuentro se analizaron las estrategias que el docente podía implementar frente a futuras dificultades de los escolares. Al igual que en el taller para padres, las docentes realizaron la correspondiente evaluación del taller.

\section{APLICACIÓN DE PROGRAMAS DE} INTERVENCIÓN: RESULTADOS OBTENIDOS

\section{Estudio 1}

En el año 2004 se trabajó con 62 niños/as de entre 7 y 11 años de edad, de nivel socioeconómico medio, que concurrían al primer y segundo ciclo de educación general básica de una escuela primaria estatal del departamento de Guaymallén, en la provincia de Mendoza, Argentina (Ison et al., 2005).

Los niños/as fueron asignados, en forma aleatoria, a dos subgrupos: experimental y control. El grupo experimental estuvo compuesto por 34 niños/as con disfunción atencional, participantes del programa de intervención. La edad media de los niños/as fue igual a 7,94 $(\mathrm{DE}=1,04)$. El grupo control estuvo conformado por 28 escolares con disfunción atencional, el cual no participó del programa de intervención. Edad media = 8,54 (DE = 1,34).

Al grupo experimental se le aplicó el test de atención infantil, programa diseñado para estimular estrategias atencionales. Consta de distintos subtest dirigidos a estimular la focalización y el sostenimiento atencional mediante tareas de búsqueda visual (Ison, Soria \& Ana, 2003).

Los talleres para docentes y padres se desarrollaron en el mismo establecimiento educativo, en encuentros programados previamente.

\section{Grupo experimental}

Como muestra la tabla 1, el grupo de niños de 7-8 años logró incrementar significativamente los puntajes de atención focalizada $(t=2.65, \mathrm{p}<.01)$ $\mathrm{y}$ atención sostenida $(t=4.75$, $\mathrm{p}<.000$ ) con posterioridad al programa de intervención. En tanto que el grupo de niños de 9-11 años logró incrementar significativamente los puntajes en atención sostenida $(t=3.26, \mathrm{p}<.01)$ pero no así en atención focalizada $(t=2.11, \mathrm{p}<.07)$, observándose solo una tendencia al incremento de las puntuaciones.

Al analizar las habilidades constructivas, evaluadas por medio del test Rey en su fase de copia, en el grupo de menor edad (7-8 años) se observó un 
incremento estadísticamente significativo en la precisión de la copia $(t=$ $2.27, \mathrm{p}<.03)$ y una disminución significativa en el tiempo empleado para realizarla $(t=2.64, \mathrm{p}<.01)$. Sin embargo, en el grupo de mayor edad (9-11 años) no se observaron diferencias estadísticamente significativas en ninguna de las dos variables consideradas: precisión y tiempo de copia.

La memoria visual no verbal fue evaluada por medio del test Rey en su fase de reproducción de memoria, que evalúa tanto la precisión del recuerdo como el tiempo empleado para su reproducción. Al comparar los resultados obtenidos con posterioridad al programa de intervención se observó que el grupo de niños menores incrementó significativamente los puntajes en la fase de reproducción de memoria $(t=2.41, \mathrm{p}<.02)$. Por el contrario, al analizar los resultados obtenidos por el grupo de niños mayores (9-11 años) se observó un incremento estadísticamente significativo en el tiempo para reproducir de memoria la gráfica $(t=3$, $\mathrm{p}<.03)$ pero no así en la precisión del recuerdo.

$\mathrm{Al}$ analizar los resultados obtenidos en cada una de las habilidades cognitivas para la solución de problemas interpersonales se observó que el grupo de niños de 7-8 años presentó un incremento estadísticamente significativo en las siguientes habilidades cognitivas: identificación de situaciones de interacción social que constituyen un pro- blema $(t=2.28, \mathrm{p}<.03)$; descripción de una situación problema $(t=4.36$, $\mathrm{p}<.000)$; generación de alternativas asertivas de solución tanto en la formulación de categorías $(t=2.56, \mathrm{p}<.01)$ como en enumeraciones $(t=2.05$, $\mathrm{p}<.05)$. Por otra parte, se observó una disminución estadísticamente significativa en la toma decisiones inadecuadas.

En el grupo de niños mayores (9-11 años) no se observaron diferencias estadísticamente significativas en ninguna de las habilidades cognitivas para la solución de problemas interpersonales con posterioridad al programa de intervención.

\section{Grupo control}

Todos los niños de la muestra presentaron puntuaciones significativamente mayores en focalización atencional al comparar los resultados obtenidos entre la primera y la segunda evaluación (edad 7-8 años, $t=3.13, \mathrm{p}<.007$; edad 9-11 años, $t=2.47, \mathrm{p}<.02)$. Sin embargo, en atención sostenida no se observaron diferencias estadísticamente significativas en ninguno de los dos rangos de edades (7-8 / 9-11 años) al comparar la primera y la segunda evaluación.

Al analizar las habilidades constructivas en ambos rangos de edades (7-8 / 9-11 años) no se observaron diferencias significativas en la precisión de la copia, pero ambos grupos obtuvieron una disminución significativa en el 
Tabla 1

Diferencia de medias intragrupo: Niños con disfunción atencional participantes del programa de intervención, por rangos de edades según atención focalizada, sostenida, habilidades constructivas y memoria visual no verbal.

\begin{tabular}{lcccccc}
\hline \multirow{2}{*}{ Grupo experimental } & \multicolumn{2}{c}{ Antes } & \multicolumn{2}{c}{ Después } & $\underline{\boldsymbol{t}}$ & $\underline{\boldsymbol{p}}$ \\
& $\mathbf{M}$ & $\mathbf{D E}$ & $\mathbf{M}$ & $\mathbf{D E}$ & & \\
\hline Atención focalizada & & & & & & \\
$\quad$ 7-8 años & .31 & .77 & .67 & .30 & 2.65 & .010 \\
9-11 años & .46 & .20 & .69 & .17 & 2.11 & .070 \\
Atención sostenida & & & & & & \\
$\quad$ 7-8 años & .36 & .14 & .50 & .13 & 4.75 & .000 \\
9-11 años & .45 & .11 & .67 & .08 & 3.26 & .010 \\
Habilidades constructivas & & & & & & \\
Puntajes 7- 8 años & 19.60 & 8 & 23.12 & 6.36 & 2.27 & .03 \\
$\quad$ Puntajes 9-11 años & 27.08 & 5.48 & 22.08 & 8.18 & 2.09 & .09 \\
$\quad$ Tiempo 7- 8 años & 6.08 & 2.32 & 4.54 & 1.28 & 2.64 & .01 \\
$\quad$ Tiempo 9-11 años & 5 & .63 & 4.83 & .98 & .30 & $\mathrm{~ns}$ \\
Memoria visual no verbal & & & & & & \\
$\quad$ Puntajes 7- 8 años & 9.89 & 5.60 & 13.60 & 6.89 & 2.41 & .02 \\
Puntajes 9-11 años & 10.58 & 6.97 & 10.41 & 6.45 & 0.04 & $\mathrm{~ns}$ \\
$\quad$ Tiempo 7- 8 años & 3.29 & 1.68 & 2.71 & 1.23 & 1.30 & $\mathrm{~ns}$ \\
$\quad$ Tiempo 9-11 años & 2 & 1.09 & 3.50 & 2.26 & 3 & .03 \\
\hline
\end{tabular}

Tabla 2

Diferencia de medias intragrupo: Niños con disfunción atencional sin la participación en el programa de intervención, por rangos de edades según atención focalizada, sostenida, habilidades constructivas y memoria visual no verbal.

\begin{tabular}{|c|c|c|c|c|c|c|}
\hline \multirow{2}{*}{ Grupo control } & \multicolumn{2}{|c|}{ Primera evaluación } & \multicolumn{2}{|c|}{ Segunda evaluación } & \multirow[t]{2}{*}{$\underline{t}$} & \multirow[t]{2}{*}{$\underline{p}$} \\
\hline & M & DE & M & DE & & \\
\hline \multicolumn{7}{|l|}{ Atención focalizada } \\
\hline 7-8 años & .49 & .42 & .73 & .32 & 3.13 & .07 \\
\hline 9-11 años & .39 & .46 & .64 & .27 & 2.47 & .02 \\
\hline \multicolumn{7}{|l|}{ Atención sostenida } \\
\hline 7-8 años & .39 & .06 & .42 & .11 & 1.33 & ns \\
\hline 9-11 años & .45 & .12 & .47 & .14 & .43 & ns \\
\hline \multicolumn{7}{|c|}{ Habilidades constructivas } \\
\hline Puntajes $7-8$ años & 19.32 & 7.62 & 20.67 & 7.05 & .53 & ns \\
\hline Puntajes $9-11$ años & 25.08 & 10.52 & 24.37 & 10.10 & .28 & ns \\
\hline Tiempo $7-8$ años & 6.64 & 2.27 & 4.32 & .77 & 3.74 & 0.002 \\
\hline Tiempo 9-11 años & 6 & 1.65 & 4 & 1.04 & 3.83 & 0.003 \\
\hline \multicolumn{7}{|c|}{ Memoria visual no verbal } \\
\hline Puntajes $7-8$ años & 7.92 & 4.71 & 9.71 & 6.05 & 1.51 & ns \\
\hline Puntajes $9-11$ años & 14.29 & 8.02 & 13.62 & 7.82 & .43 & ns \\
\hline Tiempo 7- 8 años & 4.14 & 2.41 & 2.57 & 1.02 & 2.21 & .04 \\
\hline Tiempo 9-11 años & 4.58 & 2.39 & 2.92 & 1 & 2.45 & .03 \\
\hline
\end{tabular}


tiempo empleado para realizarla (edad 7-8 años, $t=3.74, \mathrm{p}<.002$; edad 9-11 años, $t=3.83, \mathrm{p}<.003)$.

Con respecto a la memoria visual no verbal evaluada en este grupo de niños, al comparar la primera y la segunda evaluación no se observó diferencias estadísticas en la precisión del recuerdo en ninguno de los dos rangos de edades consideradas. Sin embargo, en ambos rangos de edades se observó una disminución estadísticamente significativa en el tiempo, empleado para su reproducción (edad 7-8, $t=2.21$, $\mathrm{p}<.04$; edad 9-11 años, $t=2.45$, $\mathrm{p}<.03$ ) (véase tabla 2).

$\mathrm{Al}$ analizar los resultados obtenidos por los niños de 7-8 años con disfunción atencional que no participaron del programa de intervención se observó que solo en dos de las habilidades cognitivas para la solución de problemas interpersonales hubo un incremento estadísticamente significativo: descripción de una situación problema de modo adecuado $(t=3.49, \mathrm{p}<.007) \mathrm{y}$ toma de decisión adecuada $(t=2.33$, $\mathrm{p}<.04)$. Al comparar los resultados obtenidos en la primera y la segunda evaluación, el grupo de los niños mayores (9-11 años de edad) presentó una disminución estadísticamente significativa para identificar un problema de forma adecuada $(t=3.35, \mathrm{p}<.008)$. Sin embargo, se observó un incremento estadísticamente significativo en la habilidad para tomar decisiones adecuadas $(t=2.68, \mathrm{p}<.02)$.

\section{Estudio 2}

Sobre la base de los resultados obtenidos, el 2006 se decidió enriquecer el programa de intervención aplicado previamente mediante la incorporación de una serie de estrategias destinadas a desarrollar las habilidades cognitivas de solución de problemas interpersonales, además de la atención sostenida y focalizada (Ison et al., 2007). Vale decir, el programa de intervención (tratamiento) aplicado constó de dos partes:

- Test de atención infantil 1.0, programa computarizado de entrenamiento en atención sostenida (Ison, Soria \& Ana, 2003).

- Programa para el desarrollo de habilidades cognitivas para la solución de problemas interpersonales (Ison, Morelato, Greco \& Maddio, 2006).

El programa de intervención fue aplicado dos veces por semana al grupo experimental. Para estimular la atención sostenida se aplicó el test de atención infantil en forma individual, una vez por semana, durante 14 semanas. Para estimular atención sostenida se trabajó con el test 1, que consiste en identificar si el "estímulo modelo" presentado guarda semejanzas o diferencias con otro exhibido simultáneamente en la pantalla. Los estímulos semejantes o diferentes al modelo, que aparecen en la pantalla, son 240 y se presentan en forma aleatoria. Se trabajó en una sala especialmente equipada con una PC destinada solo para este objetivo. 
En la segunda sesión semanal se aplicó, en forma grupal, el programa para desarrollar habilidades cognitivas para la solución de problemas interpersonales. Se trabajó en pequeños grupos ( 5 niños por grupo), una vez por semana, durante 9 semanas. Las sesiones de enseñanza de cada módulo duraron aproximadamente 30 minutos y estuvieron a cargo de los investigadores. También se contó con observadores no participantes, quienes registraban el comportamiento y los comentarios de los niños/as durante el desarrollo de cada sesión.

También se trabajó una vez por mes, en horas de clase, con el grupo completo de escolares con el fin de integrar al docente, brindándole herramientas psicoeducativas que lo ayudaran a implementar en su sala de clases.

Por otra parte, se realizaron talleres de capacitación para padres y docentes, con el fin de informar sobre las características de la disfunción atencional y brindar técnicas específicas para su manejo dentro del aula y en la casa. Los talleres para docentes se realizaron en forma trimestral durante 90 minutos aproximadamente. Los talleres con los padres se realizaron en forma bimestral durante 60 minutos.

Los participantes de este estudio fueron 125 escolares identificados con disfunción en atención sostenida (DAS), de ambos géneros, entre 7 y 12 años de edad, concurrentes a escuelas primarias estatales urbanas del departamento de
Guaymallén, en la provincia de Mendoza. Todos los niños pertenecieron a un nivel socioeconómico medio.

Los escolares que participaron de la muestra fueron asignados aleatoriamente a cada uno de los dos subgrupos que a continuación se describen:

- Grupo experimental: Compuesto por 67 escolares con DAS, participantes del programa de estimulación para el desarrollo de atención sostenida y habilidades cognitivas para la solución de problemas interpersonales.

- Grupo control: Conformado por 58 escolares con DAS. En este grupo de niños/as la intervención fue retrasada. En un segundo momento del proceso de investigación, vale decir después de la evaluación posterior se les administró el programa de estimulación para el desarrollo de atención sostenida y habilidades cognitivas para la solución de problemas interpersonales a fin de dar cumplimiento con las consideraciones éticas en el trabajo con seres humanos.

Con posterioridad al programa de intervención, al analizar la atención sostenida se observó que las mujeres de 7-8 años del grupo experimental lograron incrementar significativamente el número de aciertos $(t=2.22$, $\mathrm{p}<.03)$ y las puntuaciones directas $(t=2.36, \mathrm{p}<.02)$ en comparación con el grupo control (tabla 3 ). Por el con- 
trario, en el rango de edad de 9-12 años no se observaron diferencias significativas entre ambos grupos en aciertos, errores y puntuaciones directas. La diferencia observada fue en el número de omisiones realizadas por las mujeres del grupo control, quienes incrementaron significativamente sus puntuaciones en la segunda evaluación $(t=2.26, \mathrm{p}<.03)$ (véase tabla 5$)$.

En los varones, en ambos rangos de edades (7-8 y 9-12 años) no se observaron diferencias significativas entre el grupo experimental y el grupo control en ninguna de las variables consideradas en atención sostenida (aciertos, errores, omisiones y puntuación directa) (véanse tablas 4 y 6 ).

$\mathrm{Al}$ analizar las habilidades constructivas en el grupo de menor edad (7-8 años) se observó, en ambos géneros, un incremento estadísticamente significativo en la precisión de la copia y en el tiempo empleado para realizarla (tablas 3 y 4). Sin embargo, en el grupo de mayor edad (9-11 años) no se observaron diferencias estadísticamente significativas en ninguna de las dos variables consideradas: precisión y tiempo de copia (véase tablas 5 y 6 ).

Con relación a la memoria de trabajo se tuvo en cuenta tanto la precisión del recuerdo como el tiempo empleado para su reproducción. Al comparar los resultados obtenidos, con posterioridad al programa de intervención, se observó que las mujeres del grupo experimental de 7 y 8 años incrementaron sig- nificativamente el tiempo para reproducir de memoria la gráfica $(t=2.56$, $\mathrm{p}<.01)$ pero no así la precisión del recuerdo (tabla 3). Por el contrario, los varones del grupo experimental de la misma edad lograron un incremento significativo en la precisión del recuerdo de la gráfica en comparación con el grupo control $(t=1.96, \mathrm{p}<.05)$ (véase la tabla 4).

Los varones del grupo experimental de mayor edad (9 a 12 años) obtuvieron un incremento estadísticamente significativo en la precisión del recuerdo y en el tiempo empleado para su realización, en comparación con el grupo control (tabla 6). Sin embargo, en el grupo experimental de mujeres no se observaron diferencias significativas al comparar los resultados con el grupo control (véase tabla 5).

$\mathrm{Al}$ analizar los resultados obtenidos en cada una de las habilidades cognitivas para la solución de problemas interpersonales se observó que solo el grupo de varones de 7-8 años del grupo experimental presentó un incremento estadísticamente significativo en las habilidades cognitivas de solución de problemas interpersonales que se mencionan a continuación: identificación correcta de situaciones de interacción social que constituyen un problema $(t=2.45, \mathrm{p}<.02)$ y la emoción tristeza asociada a la situación problema $(t=2.14, \mathrm{p}<.04)$, anticipación de consecuencias positivas para la resolución de un problema $(t=1.98, \mathrm{p}<.05)$ y 
toma de decisiones adecuadas $(t=2.17$, $\mathrm{p}<.04)$, en comparación con el grupo control. En el grupo de niños mayores ( 9 a 11años de edad) no se observaron diferencias estadísticamente significativas en ninguna de las habilidades cognitivas para la solución de problemas interpersonales con posterioridad al programa de intervención.

\section{DISCUSIÓN}

Sobre la base de los resultados obtenidos en diversas investigaciones realizadas por nuestro equipo, este programa de intervención mostró tener mayor efectividad en los escolares de menor edad, vale decir de 7-8 años en comparación con los niños de 10-11 años. La atención sostenida, la memoria de trabajo y las habilidades cognitivas para la solución de problemas interpersonales logran mayor recuperación si su estimulación comienza a edades más tempranas.

Diversas investigaciones han mostrado que la atención, la memoria de trabajo y las habilidades cognitivas, por ejemplo la capacidad de planificación, se incrementan paulatinamente a partir de los años preescolares y el mayor avance en su desarrollo se produce entre los 7 y 10 años de edad (Pistoia et al., 2004; Romero-Ayuso et al., 2006).
Por otra parte, sabemos que la disfunción atencional, sin mediar tratamiento, puede evolucionar hacia problemas psicológicos más severos, aumentando la probabilidad de deserción escolar y exclusión del niño del sistema educacional.

El trabajo conjunto con padres y docentes más allá de brindar a estos adultos herramientas específicas para interactuar efectivamente con el niño, promueve en ellos un cambio de actitud frente a las desventajas que la disfunción ocasiona. Adultos informados sobre la problemática, responsables y comprometidos, podrán ayudar al niño a compensar sus dificultades y a ejercitar sus talentos (Korzeniowski \& Ison, 2008).

Desde esta investigación se fomenta la creación de programas preventivos, no solo terapéuticos, que favorezcan el desarrollo integral del niño y contribuyan a la creación de un clima áulico armónico (Ison et al., 2005). Por consiguiente, este modelo de abordaje psicoeducativo promueve el trabajo en equipo entre los profesionales, los padres y los docentes, con el fin de fortalecer los recursos cognitivos, afectivos $y$ sociales en el escolar y generar un clima interpersonal favorecedor de relaciones cooperativas y del desarrollo personal de cada uno de los integrantes del contexto escolar. 


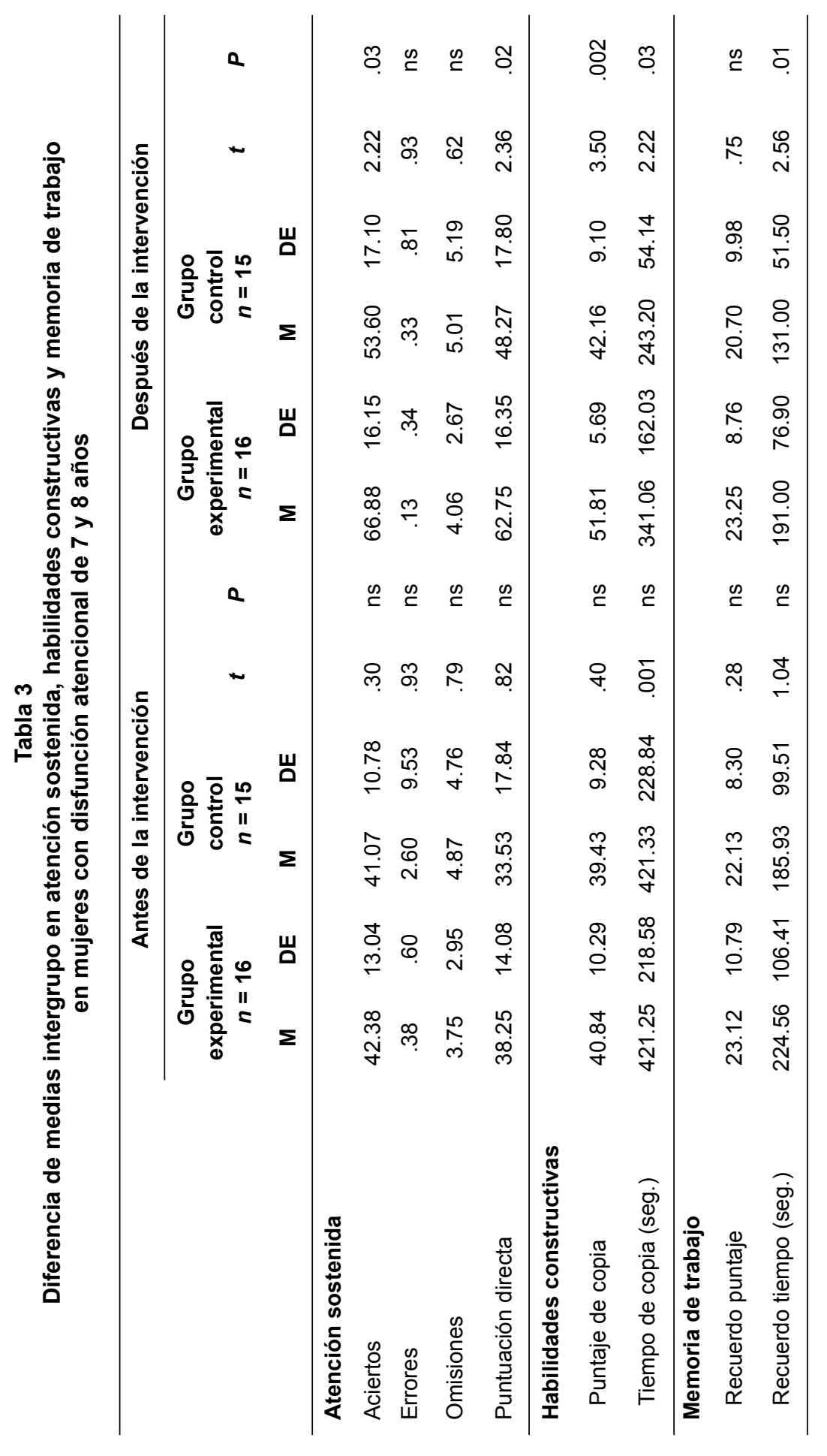




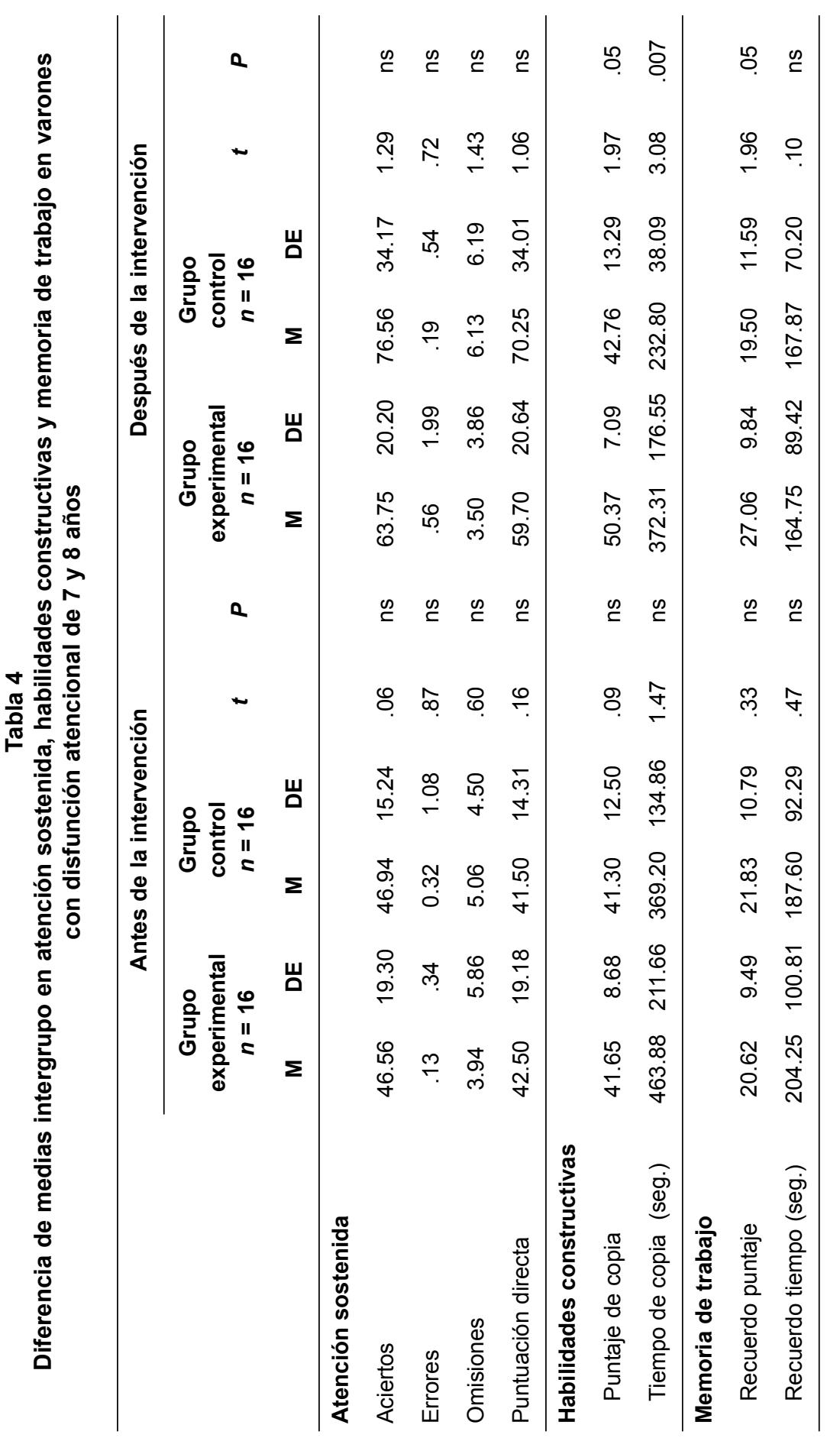




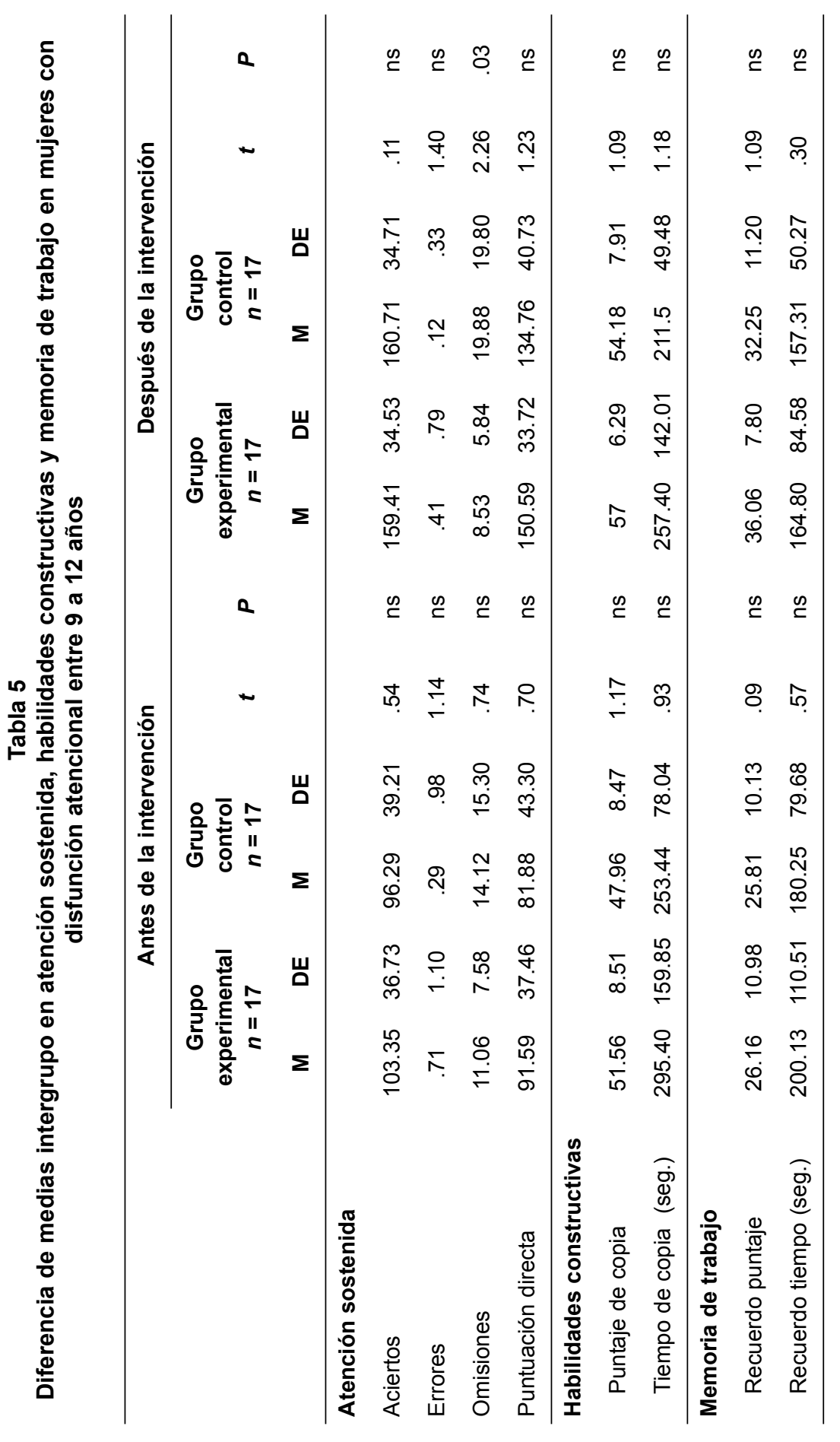




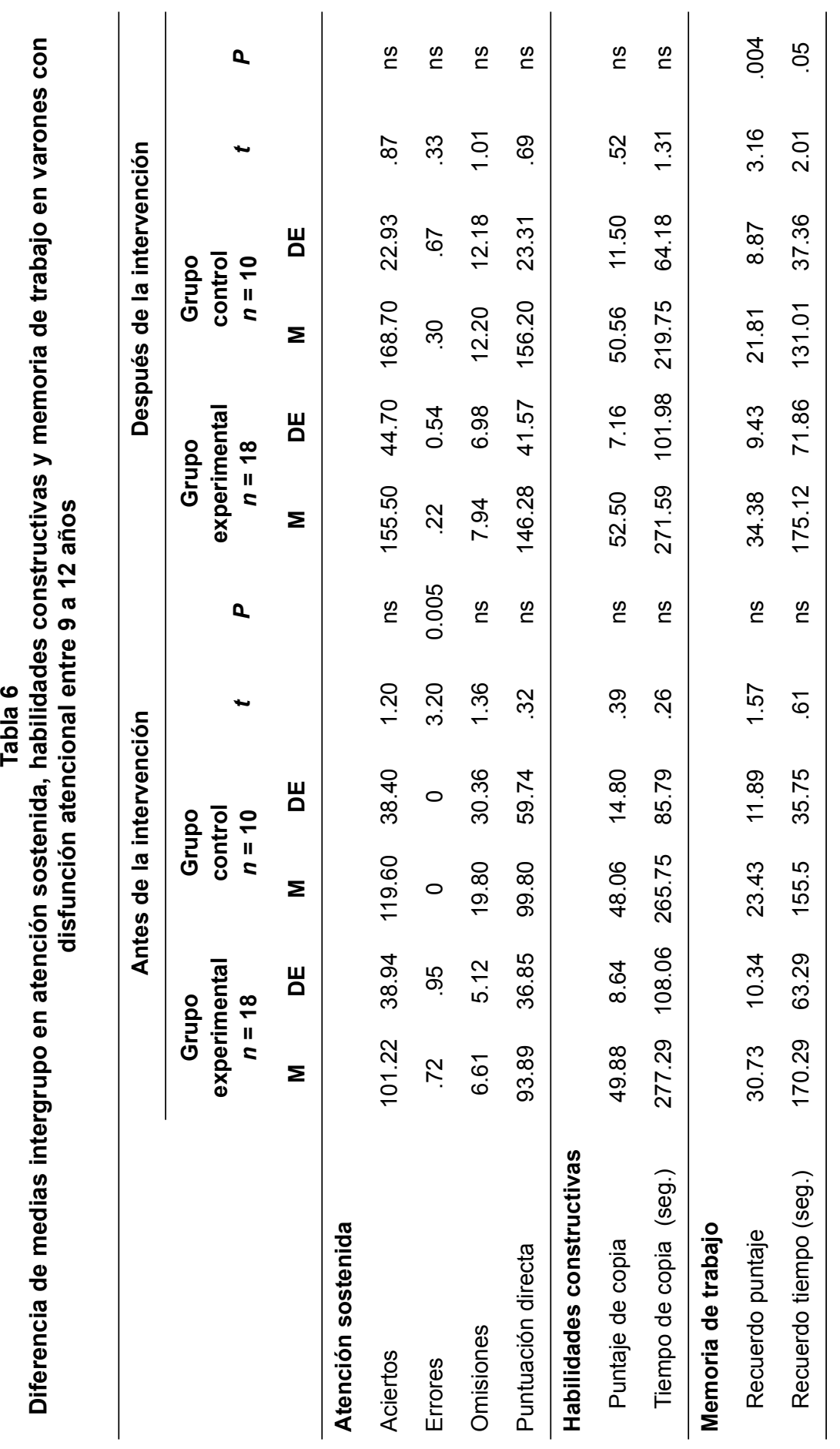




\section{REFERENCIAS}

Anderson, P. (2002). Assessment and development of executive function during childhood. Child Neuropsychol, 8, 71-82.

Baddeley, A. (1999). Memoria humana. Teoría y práctica. Madrid: McGraw-Hill.

Bauermeister, J. J. (2002). Hiperactivo, impulsivo, distraído. Guía acerca del déficit atencional para padres, maestros y profesionales. Nueva York: The Guilford Press.

Bornas, X. (1998). La autonomía personal en la infancia. Madrid: Siglo XXI.

Chugani, H. T. (1998). A critical period of brain development: Studies of cerebral glucose utilization with PET. Preventive Medicine, 27, 184-188.

García Sevilla, J. (1997). Psicología de la atención. Madrid: Síntesis.

Gauvain, M. \& Huard, R. D. (1999). Family interaction, parenting style and the development of planning: A longitudinal analysis using archival data. Journal of Family Psychology, 13, 75-92.

Ison, M. S. (2007). Estimulación de las funciones ejecutivas en escolares mendocinos. PICTO 2007-00179. Agencia Nacional de Promoción Científica y Tecnológica.
Ison, M. S. (2006). Abordaje psicoeducativo de las disfunciones atencionales en niños de edad escolar. VI Congreso Latinoamericano de Psicoterapias Cognitivas y Terapias Cognitivas en Latinoamérica: Un Paradigma en Acción. Buenos Aires, mayo del 2006.

Ison, M. S. (2004). Características familiares y habilidades socio-cognitivas en niños con conductas disruptivas. Revista Latinoamericana de Psicología, Vol. 36, (2), 257-268.

Ison, M. S. (2001a). Training in social skills: An alternative technique for handling disruptive child behavior. Perceptual and motor skills. Psychological Reports, 88, 903-911.

Ison, M. S. (2001b). Evaluación de la memoria auditiva y visual en niños hiperactivos. Interdisciplinaria 2, (18), 155-168.

Ison, M. S.; Espósito, A.; Carrada, M.; Morelato, G.; Maddio, S.; Greco, C. $\&$ Korzeniowski, C. (2007). Programa de intervención para estimular atención sostenida y habilidades cognitivas en niños con disfunción atencional. En M. C. Richaud de Minzi \& M. S. Ison (Comp.). Avances en investigación en ciencias del comportamiento en Argentina. (Tomo I). Mendoza: Universidad del Aconcagua, 115-141. 
Ison, M. S.; Soria, E. R. \& Ana, D. (2003). Test de atención infantil. Manuscrito en preparación.

Ison, M. S. \& Morelato, M. G. (2008). Habilidades socio-cognitivas en niños con conductas disruptivas y víctimas de maltrato. Universitas Psichologica, 7 (2), 357-367.

Ison, M. S.; Morelato, G.; Casals, C.; Maddio, S.; Carrada, M.; Espósito, A.; Greco, C. \& Arrigoni, F. (2005). Desarrollo de estrategias atencionales y habilidades socio-cognitivas en niños de edad escolar. En J. Vivas (Comp.). Las ciencias del comportamiento en los albores del siglo XXI. XRAACC. Mar del Plata: UNMDP, 83-97.

Ison, M. S.; Morelato, G.; Greco, C. \& Maddio, S. (2006). Programa de entrenamiento para desarrollar habilidades cognitivas de solución de problemas interpersonales. Manuscrito en preparación.

Korzeniowski, C. \& Ison, M. S. (2008). Estrategias psicoeducativas para padres y docentes de niños con TDAH. Revista Argentina de Clínica Psicológica, Vol. XVII (1), 65-71.

Lipina, S. J.; Martelli, M. I.; Vuelta, B. L.; Injoque-Ricle, I. \& Colombo, J. A. (2004). Pobreza y desempeño ejecutivo en alumnos preesolares de la ciudad de Buenos Aires. Interdisciplinaria, 21 (2), 153-193.
Muñoz-Céspedes, J. M. \& TirapuUstárroz, J. (2004). Rehabilitación de las funciones ejecutivas. Revista de Neurología, 38 (7), 656-663.

Musso, M. (2005). Funciones ejecutivas: Evaluación e intervención adecuadas al currículo escolar en una población infantil de alto riesgo. Trabajo completo publicado en el libro de la X Reunión Nacional de la la Asociación Argentina de Ciencias del Comportamiento. Junio del 2005, 129-134.

Newell, A. \& Simon, A. (1972). Human problem solving. Inglewood Cliff, NJ: Prentice-Hall.

National Institute of Child Health and Human Development. (2005). Predicting individual differences in attention, memory and planning in first graders from experiences at home, child care and school. Developmental Psychology, 41 (1), 99-114.

Ochoa Angrino, S., Cruz Panesso, I. \& Valencia, A. I. (2006). Las situaciones de resolución de problemas como estrategia de trabajo con niños diagnosticados con TDAH. Pensamiento Psicológico, 2, (7), 73-88.

Pistoia, M.; Abad-Mas, L. \& Etchepareborda, M. C. (2004). Abordaje psicopedagógico del trastorno por déficit de atención con hiperactividad con el modelo de entrenamien- 
to de las funciones ejecutivas. Revista de Neurología, 38 (Supl. 1), 149-155.

Recart-Herrera, M. I.; Mathiesen-De Gregori, M. E. \& Herrera-Garbarini, M. O. (2005). Relaciones entre algunas características de la familia del preescolar y su desempeño escolar posterior. Enfoques Educacionales, 7 (1), 105-123.

Richaud de Minzi, M. C. (2007). Fortalecimiento de recursos cognitivos, afectivos, sociales y lingüísticos en niñez en riesgo ambiental por pobreza: Un programa de intervención. En M. C. Richard \& M. S. Ison (Comp.). Avances en investigación en ciencias del comportamiento en Argentina. Mendoza: Universidad del Aconcagua.

Romero-Ayuso, D. M.; Maestú, F.; González-Marqués, J.; Romo-Barrientos, C. \& Andrade, J. M.
(2006). Disfunción ejecutiva en el trastorno por déficit de atención con hiperactividad en la infancia. Revista de Neurología, 42 (5), 265-271.

Rosselló i Mir, J. (1998). Psicología de la atención. Introducción al estudio del mecanismo atencional. Madrid: Pirámide.

Sánchez Carpintero, R. \& Narbona, J. (2004). El sistema ejecutivo y las lesiones frontales en el niño. Revista de Neurología, 39 (2), 188-191.

Tudela, P. (1992). Atención. En J. Mayor \& J. L. Pinillos (Eds.). Tratado de psicología general. Vol. 3. Atención y Percepción (pp. 11962). Madrid: Alhambra.

Zelazo, P. D.; Carter, A.; Reznick, J. S. \& Frye, D. (1997). Early development of executive function: a problem-solving framework. Review of General Psychology, 1, 198-226. 